Bull. Austral. Math. Soc.

30D 35, 30D30

Vol. 53 (1996) [71-82]

\title{
UNICITY THEOREMS FOR MEROMORPHIC
} OR ENTIRE FUNCTIONS III

\author{
HONG-XUN YI
}

This paper studies the unique range set of meromorphic functions and shows that the set $S=\left\{w \mid w^{13}+w^{11}+1=0\right\}$ is unique range set of meromorphic functions with 13 elements.

\section{INTRODUCTION}

By a meromorphic function we shall always mean a meromorphic function in the complex plane. We use the usual notations of Nevanlinna theory of meromorphic functions as explained in [4]. We use $E$ to denote any set of positive real numbers of finite linear measure, not necessarily the same at each occurrence. We denote by $S(r, f)$ any quantity satisfying $S(r, f)=o(T(r, f))(r \rightarrow \infty, r \notin E)$.

Let $f$ be a nonconstant meromorphic function and let $S$ be a subset of distinct elements in the complex plane. Define

$$
E_{f}(S)=\bigcup_{a \in S}\{z \mid f(z)-a=0\}
$$

where each zero of $f(z)-a$ with multiplicity $m$ is repeated $m$ times in $E_{f}(S)$ (see [1]).

In 1976, Gross [2] proved that there exist three finite sets $S_{j}(j=1,2,3)$ such that any two entire functions $f$ and $g$ satisfying $E_{f}\left(S_{j}\right)=E_{g}\left(S_{j}\right)$ for $j=1,2,3$ must be identical, and asked the following question (see [2, Question 6]):

Question 1. Can one find two (or possible even one) finite sets $S_{j}(j=1,2)$ such that any two nonconstant entire functions $f$ and $g$ satisfying $E_{f}\left(S_{j}\right)=E_{g}\left(S_{j}\right)$ for $j=1,2$ must be identical?

Now it is natrual to ask the following question:

Received 21 March 1995

Project supported by the National Natural Science Foundation of Peoples Republic of China.

Copyright Clearance Centre, Inc. Serial-fee code: 0004-9729/96 \$A2.00+0.00. 
Question 2. Can one find two (or possible even one) finite sets $S_{j}(j=1,2)$ such that any two nonconstant meromorphic functions $f$ and $g$ satisfying $E_{f}\left(S_{j}\right)=E_{g}\left(S_{j}\right)$ for $j=1,2$ must be identical?

Recently, the present author proved the following results which provide positive answers to Question 1.

Theorem A. (See [7, Theorem 3].) Let $S_{1}=\left\{w \mid w^{n}-1=0\right\}, S_{2}=\{a, b\}$, where $n>6$ is a positive integer, $a$ and $b$ are constants such that $a b \neq 0, a^{n} \neq b^{n}$, $a^{2 n} \neq 1, b^{2 n} \neq 1$ and $a^{n} b^{n} \neq 1$. Suppose that $f$ and $g$ are nonconstant entire functions satisfying $E_{f}\left(S_{j}\right)=E_{g}\left(S_{j}\right)$ for $j=1,2$. Then $f \equiv g$.

Theorem B. (See $\left[8\right.$, Theorem 1].) Let $S=\left\{w \mid w^{n}+a w^{n-m}+b=0\right\}$, where $n$ and $m$ are two positive integers such that $n$ and $m$ have no common factors and $n \geqslant 2 m+5, a$ and $b$ are two nonzero constants such that the algebraic equation $w^{n}+a w^{n-m}+b=0$ has no multiple roots. If $f$ and $g$ are nonconstant entire functions satisfying $E_{f}(S)=E_{g}(S)$, then $f \equiv g$.

Recently, the present author proved the following result which is a partial answer of Question 2.

Theorem C. (See [8, Theorem 2].) Let $S=\left\{w \mid w^{n}+a w^{n-m}+b=0\right\}$, where $n$ and $m$ are two positive integers such that $m \geqslant 2, n \geqslant 2 m+7$ with $n$ and $m$ having no common factors, $a$ and $b$ are two nonzero constants such that the algebraic equation $w^{n}+a w^{n-m}+b=0$ has no multiple roots. Suppose that $f$ and $g$ are nonconstant meromorphic functions satisfying $E_{f}(S)=E_{g}(S)$ and $E_{f}(\{\infty\})=E_{g}(\{\infty\})$. Then $f \equiv g$.

The set $S$ such that for any two nonconstant meromorphic functions $f$ and $g$ the condition $E_{f}(S)=E_{g}(S)$ implies $f \equiv g$ is called a unique range set (URS in brief) of meromorphic functions. A similar definition for entire functions can be given. From Theorem B we immediately obtain the following result.

ThEOREM $\mathrm{B}^{\prime}$. Let $S$ be defined as in Theorem $B$. Then $S$ is a URS of entire functions.

As a special case of Theorem $B^{\prime}$, we deduce that the set $S=\left\{w \mid w^{7}+w^{6}+1=0\right\}$ in a URS of entire functions with 7 elements. In this paper, we shall exhibit a URS of meromorphic functions with 13 elements. In fact, we prove more generally the following theorem, which provides a positive answer to Question 2.

Theorem 1. Let $S=\left\{w \mid w^{n}+a w^{n-m}+b=0\right\}$, where $n$ and $m$ are two positive integers such that $n$ and $m$ have no common factors, $m \geqslant 2$ and $n>2 m+8$, $a$ and $b$ are two nonzero constants such that the algebraic equation $w^{n}+a w^{n-m}+b=0$ has no multiple roots. Then $S$ is a URS of meromorphic functions. 
From Theorem 1 we immediately obtain that the set $S=\left\{w \mid w^{13}+w^{11}+1=0\right\}$ provides a URS of meromorphic functions with 13 elements, which provides a positive answer to Question 2.

\section{SOME LEMMAS}

LEMMA 1. (See [5].) Let $f$ be a nonconstant meromorphic function, and let $P(f)$ be a polynomial in $f$ of the form

$$
P(f)=a_{0} f^{n}+a_{1} f^{n-1}+\ldots+a_{n-1} f+a_{n}
$$

where $a_{0}(\neq 0), a_{1}, \ldots, a_{n}$ are constants. Then

$$
T(r, P(f))=n T(r, f)+S(r, f) .
$$

In order to state the second lemma, we introduce the following notation.

Let $F$ be a meromorphic function. We denote by $n_{1}(r, 1 /(F-a))$ the number of simple $a$-points of $F$ in $|z| \leqslant r . \quad N_{1}(r, 1 /(F-a))$ is defined in terms of $n_{1}(r, 1 /(F-a))$ in the usual way (see [6]).

Let $F$ and $G$ be two nonconstant meromorphic functions. If $F$ and $G$ have the same $a$-points with the same multiplicities, we say $F$ and $G$ share the value $a \mathrm{CM}$ (see $[3])$.

LEMMA 2, Let

$$
H=\left(\frac{F^{\prime \prime}}{F^{\prime}}-\frac{2 F^{\prime}}{F-1}\right)-\left(\frac{G^{\prime \prime}}{G^{\prime}}-\frac{2 G^{\prime}}{G-1}\right),
$$

where $F$ and $G$ are two nonconstant meromorphic functions. If $F$ and $G$ share $1 C M$, and $H \not \equiv 0$, then

$$
N_{1}\left(r, \frac{1}{F-1}\right) \leqslant N\left(r, \frac{1}{H}\right) \text {. }
$$

Proof: Suppose that $z_{0}$ is a simple 1-point of $F$. Let

$$
\begin{aligned}
& F(z)=1+a_{1}\left(z-z_{0}\right)+a_{2}\left(z-z_{0}\right)^{2}+O\left(\left(z-z_{0}\right)^{3}\right) \\
& G(z)=1+b_{1}\left(z-z_{0}\right)+b_{2}\left(z-z_{0}\right)^{2}+O\left(\left(z-z_{0}\right)^{3}\right)
\end{aligned}
$$

where $a_{1} \neq 0$ and $b_{1} \neq 0$. Then an elementary calculation gives that

$$
H(z)=O\left(z-z_{0}\right)
$$

which proves that $z_{0}$ is a zero of $H$. Thus,

$$
N_{1}\left(r, \frac{1}{F-1}\right) \leqslant N\left(r, \frac{1}{H}\right) \text {. }
$$




\section{Proof of Theorem 1}

Suppose that $f$ and $g$ are two nonconstant meromorphic functions satisfying $E_{f}(S)=E_{g}(S)$. We proceed to prove $f \equiv g$.

Let

$$
F=-\frac{1}{b} f^{n-m}\left(f^{m}+a\right) \quad \text { and } \quad G=-\frac{1}{b} g^{n-m}\left(g^{m}+a\right)
$$

From Lemma 1, we have

$$
T(r, F)=n T(r, f)+S(r, f)
$$

and

$$
T(r, G)=n T(r, g)+S(r, g)
$$

Let

$$
T(r)=\max \{T(r, f), T(r, g)\}
$$

and

$$
S(r)=o(T(r)) \quad(r \rightarrow \infty, r \notin E) .
$$

Noting $S=\left\{w \mid w^{n}+a w^{n-m}+b=0\right\}$, from $E_{f}(S)=E_{g}(S)$ we get that $F$ and $G$ share the value $1 \mathrm{CM}$.

Let $H$ be given by (1). If $H \not \equiv 0$, from Lemma 2 we have

$$
N_{1}\left(r, \frac{1}{F-1}\right) \leqslant N\left(r, \frac{1}{H}\right) \leqslant T(r, H)+O(1) .
$$

From (1) we obtain

$$
m(r, H)=S(r)
$$

From (2) we have

$$
F^{\prime}=-\frac{1}{b} f^{n-m-1}\left(n f^{m}+a(n-m)\right) f^{\prime}
$$

and

$$
G^{\prime}=-\frac{1}{b} g^{n-m-1}\left(n g^{m}+a(n-m)\right) g^{\prime}
$$


Since $F$ and $G$ share $1 \mathrm{CM}$, from (1), (7) and (8),

(9)

$$
\begin{aligned}
N(r, H) \leqslant & \bar{N}(r, f)+\bar{N}(r, g)+\bar{N}\left(r, \frac{1}{f}\right)+\bar{N}\left(r, \frac{1}{n f^{m}+a(n-m)}\right)+N_{0}\left(r, \frac{1}{f^{\prime}}\right) \\
& +\bar{N}\left(r, \frac{1}{g}\right)+\bar{N}\left(r, \frac{1}{n g^{m}+a(n-m)}\right)+N_{0}\left(r, \frac{1}{g^{\prime}}\right) \\
\leqslant & (m+2) T(r, f)+(m+2) T(r, g)+N_{0}\left(r, \frac{1}{f^{\prime}}\right)+N_{0}\left(r, \frac{1}{g^{\prime}}\right)+O(1),
\end{aligned}
$$

where $N_{0}\left(r, 1 / f^{\prime}\right)$ denotes the counting function corresponding to the zeros of $f^{\prime}$ that are not zeros of $f$ and $F-1, N_{0}\left(r, 1 / g^{\prime}\right)$ denotes the counting function corresponding to the zeros of $g^{\prime}$ that are not zeros of $g$ and $G-1$. It follows from (5), (6) and (9) that

$$
\begin{aligned}
N_{1}\left(r, \frac{1}{F-1}\right) \leqslant( & +2) T(r, f)+(m+2) T(r, g) \\
& +N_{0}\left(r, \frac{1}{f^{\prime}}\right)+N_{0}\left(r, \frac{1}{g^{\prime}}\right)+S(r) .
\end{aligned}
$$

Suppose that $w_{1}, w_{2}, \ldots, w_{n}$ are the distinct roots of the equation $w^{n}+a w^{n-m}+$ $b=0$. From (2) we have

$$
F-1=-\frac{1}{b}\left(f-w_{1}\right)\left(f-w_{2}\right) \ldots\left(f-w_{n}\right)
$$

and

$$
G-1=-\frac{1}{b}\left(g-w_{1}\right)\left(g-w_{2}\right) \ldots\left(g-w_{n}\right)
$$

By the second fundamental theorem, we deduce

$$
\begin{aligned}
n T(r, f) & <\bar{N}(r, f)+\bar{N}\left(r, \frac{1}{f}\right)+\sum_{j=1}^{n} \bar{N}\left(r, \frac{1}{f-w_{j}}\right)-N_{0}\left(r, \frac{1}{f^{\prime}}\right)+S(r) \\
& \leqslant 2 T(r, f)+\bar{N}\left(r, \frac{1}{F-1}\right)-N_{0}\left(r, \frac{1}{f^{\prime}}\right)+S(r) .
\end{aligned}
$$

In the same manner as above, we have

$$
n T(r, g)<2 T(r, g)+\bar{N}\left(r, \frac{1}{G-1}\right)-N_{0}\left(r, \frac{1}{g^{\prime}}\right)+S(r)
$$


It is obvious that

$$
\begin{aligned}
\bar{N}\left(r, \frac{1}{F-1}\right)+\bar{N}\left(r, \frac{1}{G-1}\right) & =2 \bar{N}\left(r, \frac{1}{F-1}\right) \\
& \leqslant N_{1}\left(r, \frac{1}{F-1}\right)+N\left(r, \frac{1}{F-1}\right) \\
& \leqslant N_{1}\left(r, \frac{1}{F-1}\right)+T(r, F)+O(1) \\
& =N_{1}\left(r, \frac{1}{F-1}\right)+n T(r, f)+S(r)
\end{aligned}
$$

and

$$
\bar{N}\left(r, \frac{1}{F-1}\right)+\bar{N}\left(r, \frac{1}{G-1}\right) \leqslant N_{1}\left(r, \frac{1}{F-1}\right)+n T(r, g)+S(r) .
$$

From (10), (13), (14) and (15) we obtain

$$
n T(r, g) \leqslant(m+4) T(r, f)+(m+4) T(r, g)+S(r) .
$$

From (10), (13), (14) and (16) we obtain

$$
n T(r, f) \leqslant(m+4) T(r, f)+(m+4) T(r, g)+S(r) .
$$

Thus,

$$
\begin{aligned}
n T(r) & \leqslant(m+4) T(r, f)+(m+4) T(r, g)+S(r) \\
& \leqslant(2 m+8) T(r)+S(r) .
\end{aligned}
$$

Since $n>2 m+8,(17)$ is a contradiction. From this we derive $H \equiv 0$. By integration we have from (1),

$$
\frac{1}{G-1}=\frac{A}{F-1}+B
$$

where $A(\neq 0)$ and $B$ are constants. Thus,

$$
G=\frac{(B+1) F+(A-B-1)}{B F+(A-B)} .
$$

From (18),

$$
T(r, G)=T(r, F)+O(1)
$$

and

$$
T(r)=T(r, f)+S(r, f) .
$$


From (2) we have

$$
\begin{aligned}
\bar{N}(r, F) & =\bar{N}(r, f) \leqslant T(r) \\
\bar{N}(r, G) & =\bar{N}(r, g) \leqslant T(r) \\
\bar{N}\left(r, \frac{1}{F}\right) & =\bar{N}\left(r, \frac{1}{f}\right)+\bar{N}\left(r, \frac{1}{f^{m}+a}\right) \leqslant(m+1) T(r)+O(1) \\
\bar{N}\left(r, \frac{1}{G}\right) & =\bar{N}\left(r, \frac{1}{g}\right)+\bar{N}\left(r, \frac{1}{g^{m}+a}\right) \leqslant(m+1) T(r)+O(1)
\end{aligned}
$$

We discuss the following three cases.

Case I. Suppose that $B \neq 0,-1$.

If $A-B-1 \neq 0$, from (18) we have

$$
\bar{N}\left(r, \frac{1}{F+\frac{A-B-1}{B+1}}\right)=\bar{N}\left(r, \frac{1}{G}\right) .
$$

From this and the second fundamental theorem, we have

$$
\begin{aligned}
T(r, F) & <\bar{N}(r, F)+\bar{N}\left(r, \frac{1}{F}\right)+\bar{N}\left(r, \frac{1}{F+\frac{A-B-1}{B+1}}\right)+S(r, F) \\
& =\bar{N}(r, F)+\bar{N}\left(r, \frac{1}{F}\right)+\bar{N}\left(r, \frac{1}{G}\right)+S(r, F) .
\end{aligned}
$$

Combining this with (3), (19), (20), (22) and (23), we obtain

$$
n T(r)<(2 m+3) T(r)+S(r)
$$

which contradicts the assumption $n>2 m+8$. Thus $A-B-1=0$. From (18),

$$
G=\frac{(B+1) F}{B F+1}
$$

From this we have

$$
\bar{N}\left(r, \frac{1}{F+\frac{1}{B}}\right)=\bar{N}(r, G) .
$$

Again from the second fundamental theorem, we obtain

$$
\begin{aligned}
T(r, F) & <\bar{N}(r, F)+\bar{N}\left(r, \frac{1}{F}\right)+\bar{N}\left(r, \frac{1}{F+\frac{1}{B}}\right)+S(r, F) \\
& =\bar{N}(r, F)+\bar{N}\left(r, \frac{1}{F}\right)+\bar{N}(r, G)+S(r, F) .
\end{aligned}
$$

Combining this with (3), (19), (20), (21) and (22), we obtain

$$
n T(r)<(m+3) T(r)+S(r)
$$

which is impossible. 
CAsE II. Suppose that $B=-1$.

From (18) we have

$$
G=\frac{A}{-F+(A+1)} .
$$

If $A+1 \neq 0$, from (24) we obtain

$$
\bar{N}\left(r, \frac{1}{F-(A+1)}\right)=\bar{N}(r, G) .
$$

Thus, in the same manner as above, we have a contradiction. From this we obtain $A+1=0$. Again from (24) we derive $F \cdot G \equiv 1$. This and (2) yield

$$
f^{n-m}\left(f-a_{1}\right)\left(f-a_{2}\right) \ldots\left(f-a_{m}\right) g^{n-m}\left(g^{m}+a\right) \equiv b^{2},
$$

where $a_{1}, a_{2}, \ldots, a_{m}$ are the distinct roots of the equation $\omega^{m}+a=0$.

Suppose that $z_{0}$ is a zero of $f$ of order $p$. From (25) we know that $z_{0}$ is a pole of $g$. Suppose that $z_{0}$ is a pole of $g$ of order $q$. From (25) we obtain

$$
(n-m) p=n q
$$

Noting that $n$ and $m$ have no common factors, from (26) we get $n \leqslant p$. Thus,

$$
\bar{N}\left(r, \frac{1}{f}\right) \leqslant \frac{1}{n} N\left(r, \frac{1}{f}\right) \leqslant \frac{1}{n} T(r, f)+O(1) .
$$

Suppose that $z_{j}(j=1,2, \ldots, m)$ is a zero of $f-a_{j}$ of order $p_{j}$. From (25) we know that $z_{j}$ is a pole of $g$. Suppose that $z_{j}$ is a pole of $g$ of order $q_{j}$. From (25) we obtain

$$
p_{j}=n q_{j}
$$

Thus $n \leqslant p_{j}$ and hence

$$
\bar{N}\left(r, \frac{1}{f-a_{j}}\right) \leqslant \frac{1}{n} N\left(r, \frac{1}{f-a_{j}}\right) \leqslant \frac{1}{n} T(r, f)+O(1) .
$$

By the second fundamental theorem, from (27) and (28) we have

$$
\begin{aligned}
(m-1) T(r, f) & <\bar{N}\left(r, \frac{1}{f}\right)+\sum_{j=1}^{m} \bar{N}\left(r, \frac{1}{f-a_{j}}\right)+S(r, f) \\
& \leqslant \frac{m+1}{n} T(r, f)+S(r, f),
\end{aligned}
$$

which is impossible. 
CASE III. Suppose that $B=0$.

From (18) we have

$$
G=\frac{F+(A-1)}{A}
$$

If $A-1 \neq 0$, from (29) we obtain

$$
\bar{N}\left(r, \frac{1}{F+(A-1)}\right)=\bar{N}\left(r, \frac{1}{G}\right)
$$

Thus, in the same manner as above, we have a contradiction. From this we obtain $A-1=0$. Again from (29) we derive $F \equiv G$. This and (2) yield

$$
f^{n}-g^{n}=-a\left(f^{n-m}-g^{n-m}\right)
$$

If $f^{n} \not \equiv g^{n}$, from (30) we obtain

$$
g^{m}=-\frac{a(h-v)\left(h-v^{2}\right) \ldots\left(h-v^{n-m-1}\right)}{(h-u)\left(h-u^{2}\right) \ldots\left(h-u^{n-1}\right)}
$$

where $h=f / g, u=\exp ((2 \pi i) / n)$ and $v=\exp ((2 \pi i) /(n-m))$. From (31) we know that $h$ is a nonconstant meromorphic function. Since $n$ and $m$ have no common factors, we have $u^{j} \neq v^{k}(j=1,2, \ldots, n-1 ; k=1,2, \ldots, n-m-1)$. Suppose that $z_{j}(j=1,2, \ldots, n-1)$ is a zero of $h-u^{j}$ of order $p_{j}$. From (31) we have $p_{j} \geqslant m$. Thus

$$
\bar{N}\left(r, \frac{1}{h-u^{j}}\right) \leqslant \frac{1}{m} N\left(r, \frac{1}{h-u^{j}}\right) \leqslant \frac{1}{2} T(r, h)+O(1) .
$$

By the second fundamental theorem, from (32) we obtain

$$
\begin{aligned}
(n-3) T(r, h) & <\sum_{j=1}^{n-1} \bar{N}\left(r, \frac{1}{h-u^{j}}\right)+S(r, h) \\
& \leqslant \frac{n-1}{2} T(r, h)+S(r, h),
\end{aligned}
$$

which is impossible. Thus $f^{n} \equiv g^{n}$ and $f^{n-m} \equiv g^{n-m}$. However, since $n$ and $m$ have no common factors, we get $f \equiv g$.

This completes the proof of Theorem 1 . 


\section{SuPplement of Theorem 1}

It is reasonable to ask: What can be said if $m=1$ in Theorem 1 ? In this section, we prove the following theorem, which is a supplement of Theorem 1 .

Theorem 2. Let $S=\left\{w \mid w^{n}+a w^{n-1}+b=0\right\}$, where $n>10$ is a positive integer, $a$ and $b$ are two nonzero constants such that the algebraic equation $w^{n}+a w^{n-1}+b=0$ has no multiple roots. If $f$ and $g$ are two distinct nonconstant meromorphic functions satisfying $E_{f}(S)=E_{g}(S)$, then

$$
f=-\frac{a h\left(h^{n-1}-1\right)}{h^{n}-1} \quad \text { and } \quad g=-\frac{a\left(h^{n-1}-1\right)}{h^{n}-1}
$$

where $h$ is a nonconstant meromorphic function.

Proof: Let

$$
F=-\frac{1}{b} f^{n-1}(f+a) \quad \text { and } \quad G=-\frac{1}{b} g^{n-1}(g+a) .
$$

Proceeding as in the proof of Theorem 1, we have $F \cdot G \equiv 1$ or $F \equiv G$. We distinguish the following two cases.

CAse I. Assume $F \cdot G \equiv 1$.

From (33) we have

$$
f^{n-1}(f+a) g^{n-1}(g+a) \equiv b^{2} .
$$

Suppose that $z_{0}$ is a zero of $f$ of order $p$. From (34) we know that $z_{0}$ is a pole of $g$. Suppose that $z_{0}$ is a pole of $g$ of order $q$. From (34) we obtain $(n-1) p=n q$. From this we get $n \leqslant p$. Thus

$$
\bar{N}\left(r, \frac{1}{f}\right) \leqslant \frac{1}{n} N\left(r, \frac{1}{f}\right) \leqslant \frac{1}{n} T(r, f)+O(1) .
$$

Suppose that $z_{1}$ is a zero of $f+a$ of order $p_{1}$. From (34) we know that $z_{1}$ is a pole of $g$. Suppose that $z_{1}$ is a pole of $g$ of order $q_{1}$. From (34) we obtain $p_{1}=n q_{1}$. Thus $n \leqslant p_{1}$ and hence

$$
\bar{N}\left(r, \frac{1}{f+a}\right) \leqslant \frac{1}{n} N\left(r, \frac{1}{f+a}\right) \leqslant \frac{1}{n} T(r, f)+O(1) .
$$

In the same manner as above, we have

$$
\begin{aligned}
\bar{N}\left(r, \frac{1}{g}\right) & \leqslant \frac{1}{n} T(r, g)+O(1), \\
\bar{N}\left(r, \frac{1}{g+a}\right) & \leqslant \frac{1}{n} T(r, g)+O(1) .
\end{aligned}
$$


From (34) one sees easily that the poles of $f$ can only be from the zeros of $g$ and $g+a$. Consequently,

$$
\bar{N}(r, f) \leqslant \bar{N}\left(r, \frac{1}{g}\right)+\bar{N}\left(r, \frac{1}{g+a}\right) .
$$

From this, (37) and (38) we obtain

$$
\bar{N}(r, f) \leqslant \frac{2}{n} T(r, g)+O(1) .
$$

By the first fundamental theorem and Lemma 1, from (34) we have

$$
T(r, g)=T(r, f)+S(r, f) .
$$

From this and (39) we obtain

$$
\bar{N}(r, f) \leqslant \frac{2}{n} T(r, f)+S(r, f) .
$$

By the second fundamental theorem, from (35), (36) and (40) we get

$$
\begin{aligned}
T(r, f) & <\bar{N}\left(r, \frac{1}{f}\right)+\bar{N}\left(r, \frac{1}{f+a}\right)+\bar{N}(r, f)+S(r, f) \\
& \leqslant \frac{4}{n} T(r, f)+S(r, f),
\end{aligned}
$$

which is impossible.

CASE II. Assume $F \equiv G$.

From (33) we have

$$
f^{n}-g^{n} \equiv-a\left(f^{n-1}-g^{n-1}\right)
$$

Noting $\int \not \equiv g$, from (41) we obtain

$$
g=-\frac{a\left(h^{n-1}-1\right)}{h^{n}-1}
$$

where $h=f / g$. From (42) we know that $h$ is a nonconstant meromorphic function. Thus, from (42) we have

$$
f=-\frac{a h\left(h^{n-1}-1\right)}{h^{n}-1} .
$$

This completes the proof of Theorem 2. 


\section{REFERENCES}

[1] F. Gross, 'On the distribution of values of meromorphic functions', Trans. Amer. Math. Soc. 131 (1968), 199-214.

[2] F. Gross, 'Factorization of meromorphic functions and some open problems', in Complex analysis, Proc. Conf. Univ. Kentucky, Lexington, Kentucky 1976, Lecture Notes in Math. 599 (Springer-Verlag, Berlin, Heidelberg, New York, 1977).

[3] G.G. Gundersen, 'Meromorphic functions that share three or four values', J. London Math. Soc. 20 (1979), 457-466.

[4] W.K. Hayman, Meromorphic functions (Clarendon Press, Oxford, 1964).

[5] C.C. Yang, 'On deficiencies of differential polynomials II', Math. Z. 125 (1972), 107-112.

[6] Hong-Xun Yi, 'Meromorphic functions that share two or three values', Kodai Math. J. 13 (1990), 363-372.

[7] Hong-Xun Yi, 'Unicity theorems for meromorphic or entire functions', Bull. Austral. Math. Soc. 49 (1994), 257-265.

[8] Hong-Xun $\mathrm{Yi}$, 'Unicity theorems for meromorphic or entire functions II', Bull. Austral. Math. Soc. 52 (1995), 215-224.

Department of Mathematics

Shandong University

Jinan

Shandong 250100

People's Republic of China 